\title{
EU-Vorschlag zu Künstlicher Intelligenz muss aus Datenschutz- Perspektive durchleuchtet werden
}

\section{Kugelmann: Schlupflöcher beim Einsatz von Gesichtserkennungs-Software schließen}

Die Europäische Kommission hat einen Verordnungsentwurf zur Regulierung von Künstlicher Intelligenz (KI) vorgelegt. Die angedachte EU-Verordnung wird Rheinland-Pfalz direkt betreffen.

Der Landesbeauftragte für den Datenschutz und die Informationsfreiheit (LfDI) Rheinland-Pfalz, Professor Dieter Kugelmann, sagt: „Der Einsatz von KI-Systemen ist das Zukunftsthema der 2020er und 2030er Jahre: Wie KI-Systeme programmiert, mit Daten gefüttert und eingesetzt werden, wird mit darüber bestimmen, inwieweit Freiheitsrechte innerhalb der Europäischen Union gesichert werden können. Wenn die EU ein überzeugendes Regelwerk verabschiedet, das Bürgerrechts-Bedenken ernstnimmt und gleichzeitig den technologischen Wandel nicht ausbremst, kann Europa zu einem Treiber technologischer Entwicklungen werden. Dass die EU nun anders als China und die USA voranschreitet und einen umfassenden Regulierungsentwurf vorlegt, ist durchweg zu begrüßen. Wer den Einsatz automatisierter Systeme in einer freiheitlichen Gesellschaft etablieren möchte, muss europaweit klare Regeln festzurren.“

Kugelmann sagt: „Das Verbot des Einsatzes von KI zur Massenüberwachung in dem Verordnungsvorschlag ist zu begrüßen, aber es sollte geschärft werden. Technologien zur automatisierten Gesichtserkennung sollen in der EU unter bestimmten Voraussetzungen tatsächlich zulässig sein. Der Entwurf sieht Ausnahmen für den Einsatz entsprechender Gesichtserkennungs- und Stimmerkennungssoftware vor, obwohl gerade in diesem Bereich ein besonders großes Missbrauchspotenzial besteht. Auch Systeme zur Vorratsdatenspeicherung, die sich auf den staatlichen Bereich beziehen, sollten meiner Meinung nach gänzlich untersagt werden. In der EU besteht aufgrund der europäischen Geschichte eine große Ablehnung gegenüber staatlicher Massenüberwachung."

„Andere KI-Systeme (etwa zur Auswahl von Bewerberinnen und Bewerbern in Stellenbesetzungsverfahren oder zur Bewertung der Kreditwürdigkeit von Personen), die ein hohes Missbrauchsrisiko mit sich bringen, sind nach dem Entwurf der EU-Kommission erlaubt; dies ist nachvollziehbar und die hierfür angedachten begrenzenden Regelungen sind sinnvoll. Aller- dings sollte intensiver geprüft werden, ob diese verschärften Regeln nicht auch für andere Bereiche gelten sollten, beispielsweise für Gesundheitsanwendungen“, betont Kugelmann. „KI-Systeme sollten aus meiner Sicht besonders sensible Daten - etwa genetische oder biometrische Daten sowie Gesundheitsdaten (gemäß Artikel 9 Datenschutz-Grundverordnung) - nicht standardisiert durchleuchten und in bestimmten Anwendungsfällen zu einer Überwachung nutzen können.“

Kugelmann sagt: „Die Ausgestaltung der KI-Verordnung wird unmittelbaren Einfluss auf Rheinland-Pfalz haben: Zum einen setzen vor allem größere Unternehmen wie John Deere und BASF bereits auf Künstliche Intelligenz. An welchen Stellen sie in $\mathrm{Zu}$ kunft neue Wege beschreiten, wird auch von den rechtlichen Rahmenbedingungen innerhalb der EU abhängen. Zum anderen wird in Rheinland-Pfalz vielfältige KI-Forschung betrieben - am Deutschen Forschungszentrum für künstliche Intelligenz (DFKI) in Kaiserslautern, dem Fraunhofer-Institut für Technound Wirtschaftsmathematik (ITWM), dem Fraunhofer-Institut für Experimentelles Software Engineering (IESE) sowie an den verschiedenen Universitäten und Hochschulen.“

Bis zur Verabschiedung der Verordnung steht nun ein ordentliches Gesetzgebungsverfahren an, bei dem das Europäische Parlament und die Mitgliedstaaten beteiligt sind. Der Europäische Datenschutzbeauftragte und der Europäische Datenschutzausschuss haben eine gemeinsame Stellungnahm angekündigt. Der LfDI Rheinland-Pfalz wird die Debatte konstruktiv begleiten und sich im Gesetzgebungsverfahren einbringen, etwa über die Taskforce „Künstliche Intelligenz “, welche die Konferenz der unabhängigen Datenschutzaufsichtsbehörden des Bundes und der Länder gebildet hat.

Der LfDI Rheinland-Pfalz hat während seines DSK-Vorsitzes 2019 eine „Hambacher Erklärung zur Künstlichen Intelligenz“ initiiert, die mit breiter Mehrheit verabschiedet wurde. Darin sind sieben „Datenschutzrechtliche Anforderungen an Künstliche Intelligenz" versammelt. Die Erklärung liegt in deutscher Sprache und in englischer Sprache vor.

\section{Professor Dieter Kugelmann, Der LfDI Rheinland-Pfalz}

